\title{
SPECTRAL CHARACTERIZATION OF MACRO-HETEROCYCLIC COMPOUND RhTMPyP / ZnTSPc
}

\author{
Liviu OLTEANU ${ }^{1,4}$, Rodica Mariana ION ${ }^{1,2,3}$, Sofia TEODORESCU ${ }^{4}$, \\ Raluca Maria STIRBESCU ${ }^{4}$ \\ ${ }^{1}$ Valahia University of Targoviste, Doctoral School of Materials Engineering, 130105, Targoviste, Romania \\ ${ }^{2}$ Valahia University of Targoviste, Materials Engineering and Mechanics Department, 130004, Targoviste, \\ Romania \\ ${ }^{3}$ National Institute for Research and Development in Chemistry and Petrochemistry - ICECHIM, \\ "Conservation and Evaluation of Cultural Haritage" Research Group, 060021, Bucharest, Romania \\ ${ }^{4}$ Valahia University of Targoviste, Institute of Multidisciplinary Research for Science and Technology, 130004, \\ Targoviste, Romania \\ e-mail: olteanu.liviu@icstm.ro
}

\begin{abstract}
Macro-heterocyclic compounds, such as porphyrins and phthalocyanines, are being studied extensively for their important physical and chemical properties. Their ability to absorb light throughout the spectrum and self-organization being adequate for the realization of several applications. Investigations of the spectral properties of the supramolecular assembly RhTMPyP/ZnTSPc- 5, 10,15,20 rhodium tetramethyl-pyridyl porphyrin / 2,9,16,23 zinc tetrasulfonated-phthalocyanine are registered by UV-Vis, FT-IR and Raman spectroscopic techniques.
\end{abstract}

KEYWORDS: porphyrins, phtalocyanines, macro-heterocyclic compound, UV-Vis, FT-IR, Raman

\section{Introduction}

Tetrapyrrole macrocycle based $\pi$-conjugated systems, such as porphyrin and phthalocyanine derivatives, are very famous rigid and aromatic molecular building blocks with special electronic structures [1]. Both porphyrin and phthalocyanine derivatives have distinctive photophysical, photochemical and electrochemical properties and the application of these systems have been developed in the field of photochemistry and electrochemistry [2].

As very chemically versatile compounds, both of these chemical compounds could insert different central metals, a wide range of photophysical, photochemical properties and also interesting applications could be obtained [3]. By coupling porphyrins with phthalocyanines, heterocomplexes useful for photodynamic applications could be obtained. Their association is based on „electrostatic”mixed dimers by pairing porphyrin and phtalocyanine bearing substituents with opposite charges, and have higher efficiency and stability [4].

Porphyrins are a class of macro-heterocyclic organic compounds, composed of four modified pyrrole subunits interconnected at their $\alpha$ carbon atoms via methine bridges [5]. In metallo-porphyrins, different metals (with bi-, tri- or tetra-valence) could be inserted into the macrocycle and for their spatial stability, halogen atoms could be attached as axially ligand, as is the case of Rh(III)TMPyP (Figure 1), where a single chlorine atom is necessary for this metallo-porphyrin stability [6].

Because $\mathrm{Rh}$ has a large atomic radius $(1.4 \AA)$, it cannot be included in the plane structure of porphyrin, where the macrocycle has the size of the cavity $1.3 \AA$ and, in this sense, it can adopt a position above the porphyrin plane, with chlorine as axial ligand.

Phthalocyanines are azaporphyrins consisting of four benzoindole nuclei connected by nitrogen bridges in a 16-membered ring of alternating carbon and nitrogen atoms around a central metal atom which form stable chelates with metal cations (die or paramagnetic ion).

The molecule consists of the central metal atom, which is surrounded by four nitrogen atoms-pyrrole; four other nitrogen atoms-bridging aza; 32 carbon atoms-the pyrrole and the benzene ones (Figure 2) [7, 8]. 


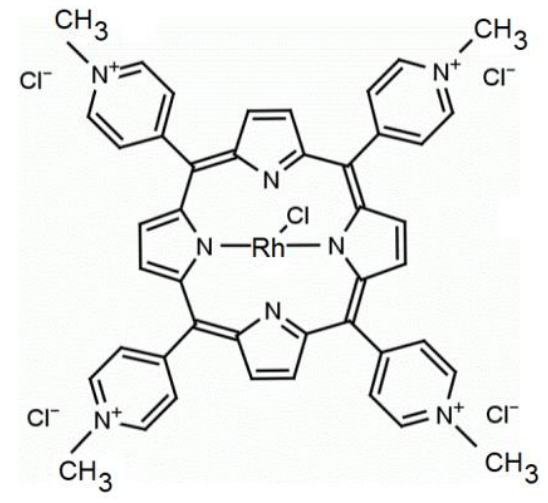

Fig. 1. Chemical structure of RhTMPyP

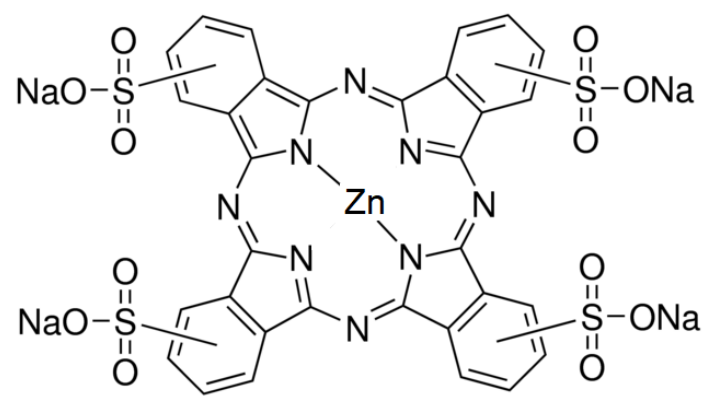

Fig. 2. Chemical structures of ZnTSPc

One of the most important properties of the porphyrin and phthalocyanine molecules is their ability to coordinate to metal ions, yielding to stable systems.

Stable complexes of metallo-porphyrins and metallo-phthalocyanines results from formation of four equivalent $\sigma$ bonds $\mathrm{N} \rightarrow \mathrm{M}$. Both of them contain a $18 \pi$ electron system that contributes to their remarkable thermal and chemical stability $[9,10]$.

In this work, the spectral properties of the macro-heterocyclic compound: rhodium-tetramethylpyridyl porphyrin (RhTMPyP) with zinc-tetrasulphonated phthalocyanine (ZnTSPc) has been studied through UV-Vis, FT-IR and Raman analysis.

\section{Materials and Methods}

\subsection{Materials}

Rh (III)TMPyP and ZnTSPc have been used as pure materials, without any supplementary procedures from Aldrich company, while their heteroaggregate has been prepared in the laboratory after the following method: Rh (III)TMPyP has been converted into their chloride salts by passing them through the VIONIT AS-14 ion-exchange column in the chloride form. Then, the phthalocyanine was transformed into its sodium salt. A $0.088 \mathrm{~g}$ sample of
ZnTSPc was dissolved on a stoichiometric amount (1:4) of $\mathrm{NaOH}(3.9 \mathrm{~mL}, 0.1 \mathrm{M})$. The solution was filtrated, and the filtrate was dried under a stream of air overnight. A $0.05 \mathrm{~g}$ amount of this product was dissolved in $30 \mathrm{~mL}$ of water.

In another beaker, an equimolar mass of $\mathrm{Rh}$ (III)TMPyP $(0.05 \mathrm{~g})$ was dissolved in the same amount of water and the two solutions were mixed.

The final solutions were left for three weeks to ensure complete precipitation and then filtered by gravity [11].

\subsection{Methods}

The UV-VIS spectra were recorded with a SPECORD M 400 Carl Zeiss Jean spectrophotometer with microprocessor and double beam.

Infrared spectra were measured using an FT-IR spectrometer (VERTEX 80), in the following conditions: range $4000 \mathrm{~cm}^{-1}$ to $580 \mathrm{~cm}^{-1}, 32$ scan, resolution $4 \mathrm{~cm}^{-1}$. FT-IR spectra were achieved using Attenuated Total Reflectance (ATR) accessory with diamond crystal. ATR spectroscopy usually requires little to no sample preparation.

Raman spectra were recorded with a portable Xantus-2 TM Raman analyzer (Rigaku, Boston, United States of America) equipped with two laser sources (i.e., $785 \mathrm{~nm}$ and $1064 \mathrm{~nm}$ ) and two detectors (i.e., TE cooled CCD and TE cooled InGaAs).

\section{Results and discussions}

Porphyrins and phthalocyanines, are compounds with great potential for serving as components of molecular materials that possess unique electronic, magnetic and photophysical properties, due to a large number of these chromophores, and for this reason it is of importance to construct arrays in which the molecules are organized in well-defined geometries with respect to their neighbours.

The phthalocyanine exhibit UV-Vis absorption spectra with an intense $\pi-\pi$ transitions, usually referred to as Q-bands, in the range 660-799 nm with associated higher energy vibrational components in the range 600-660 $\mathrm{nm}$ [12].

Also, the phthalocyanines have a B Soret band in the range 420-320 nm, an N-band around 330-285 $\mathrm{nm}$ and an L-band at $270-230 \mathrm{~nm}$. The UV-Vis spectra of the phthalocyanines are highly solvent and concentration-dependent. At high concentration in liquid protic solvent in water, for example, phthalocyanines could form aggregates [11].

During this study the spectra of phthalocyanine and porphyrin were carried out in DMSO solutions because DMSO prevents aggregation of each entity. 
In general, the UV-VIS spectra of phthalocyanine free base shows a $\mathrm{Q}$ absorption band at $718 \mathrm{~nm}$ with a shoulder at $689 \mathrm{~nm}$. As metallic complex with $\mathrm{Zn}$, and with sulphonated substituents, the phthalocyanine shows blue shifts [13].

In the porphyrin free base spectra, could be registered a Soret band (around $414 \mathrm{~nm}$ ) and four Q bands from 500 to $700 \mathrm{~nm}$.

By metallation, the Soret band support a red shift, while the $\mathrm{Q}$ bands are reduced to only two bands (around 560 and $650 \mathrm{~nm}$ ).
The existence of heterodimers and heterotrimers between the porphyrin and the phthalocyanine has been demonstrated by titration using spectrophotometric methods, such as absorption spectroscopy.

The heteroaggregate between Rh(III)TMPyP and ZnTSPc can adopt a close-contact, face-to-face mutual configuration, in order to optimize the $\pi-\pi$ interactions between aromatic cores. The spectral evolution of a solution of Rh(III)TMPyP in DMSO upon addition of ZnTSPc is shown in Figure 3.

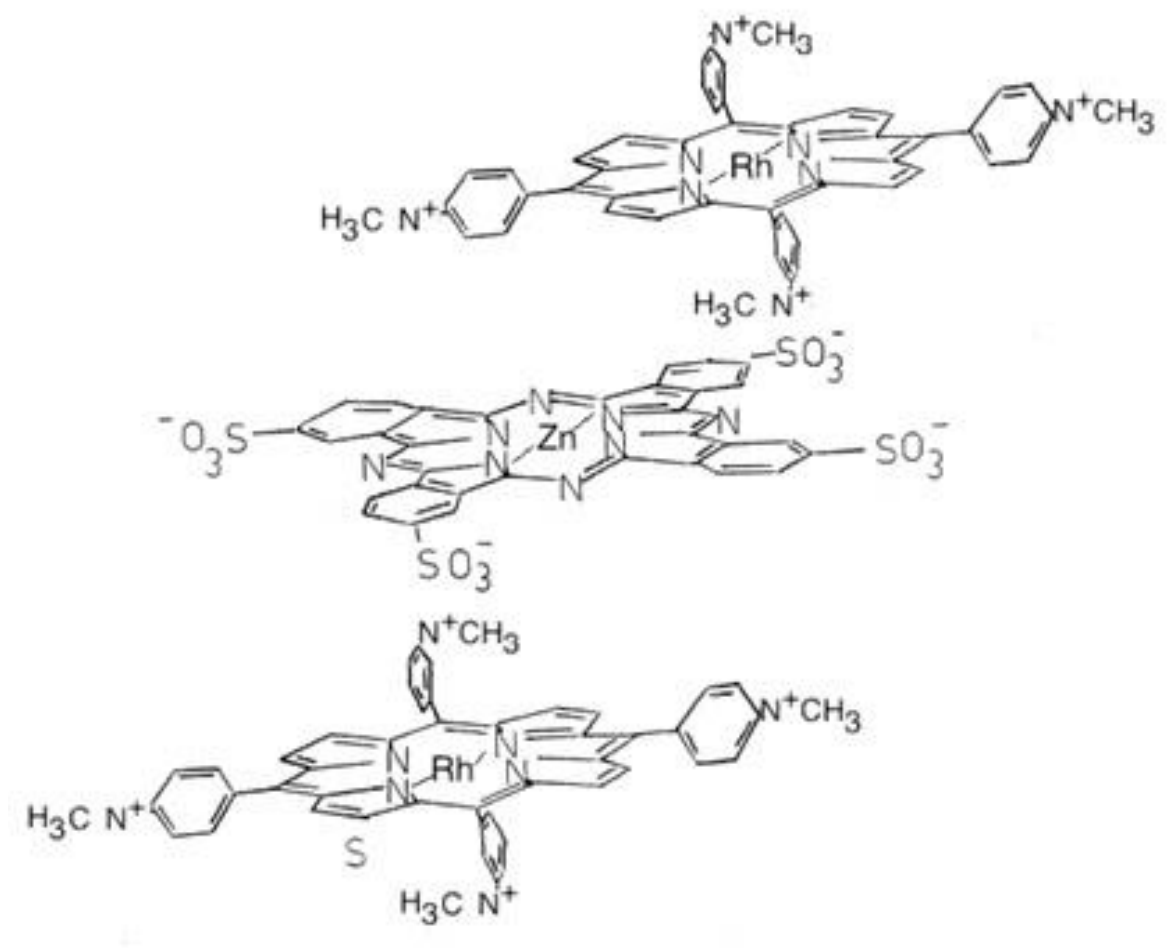

Fig. 3. The structure of the heteroaggregate Rh(III)TMPyP/ZnTSPc

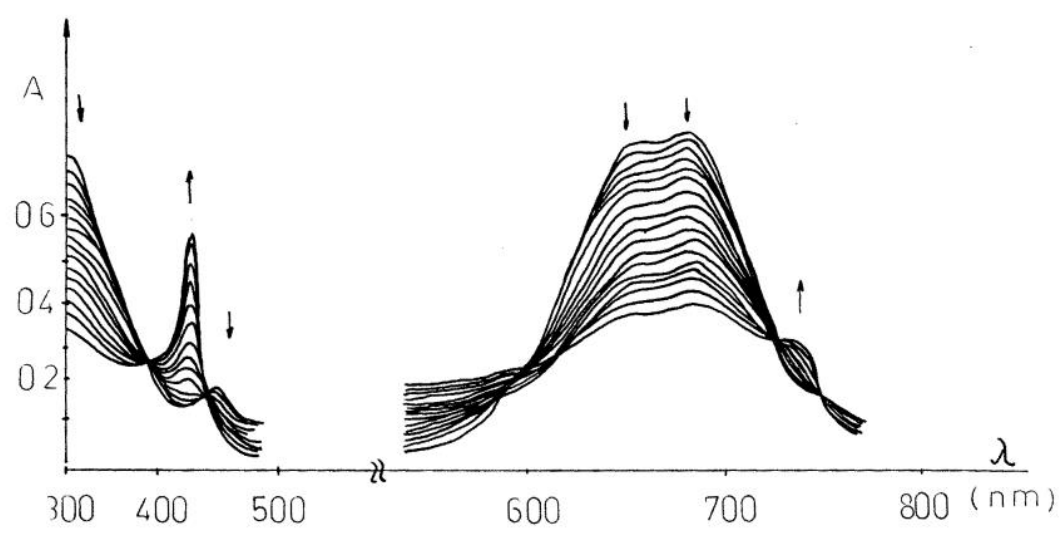

Fig. 4. The spectral changes of UV-Vis for Rh(III)TMPyP/ZnTSPc 
During the titration, the Q-band of the phthalocyanine from $717 \mathrm{~nm}$ will become less intense and broader until its disappearance. A heteroaggregate with shorter distances between the components exhibits a larger shift of the porphyrinic Q 0,0 -band.

The heteroaggregation will occur only between $\mathrm{N}+$ substituents from the porphyrine and the sulfonated phtalocyanine from the meso-positions (Figure 4).
Infrared (IR) spectroscopy is the most suitable method for identifying the presence of polar functional groups in the molecule structure of organic compounds.

To determine the structure of organic compounds, only the middle IR domain is used, and the characterization is achieved by using wavenumbers in the range $400-4000 \mathrm{~cm}^{-1}$.

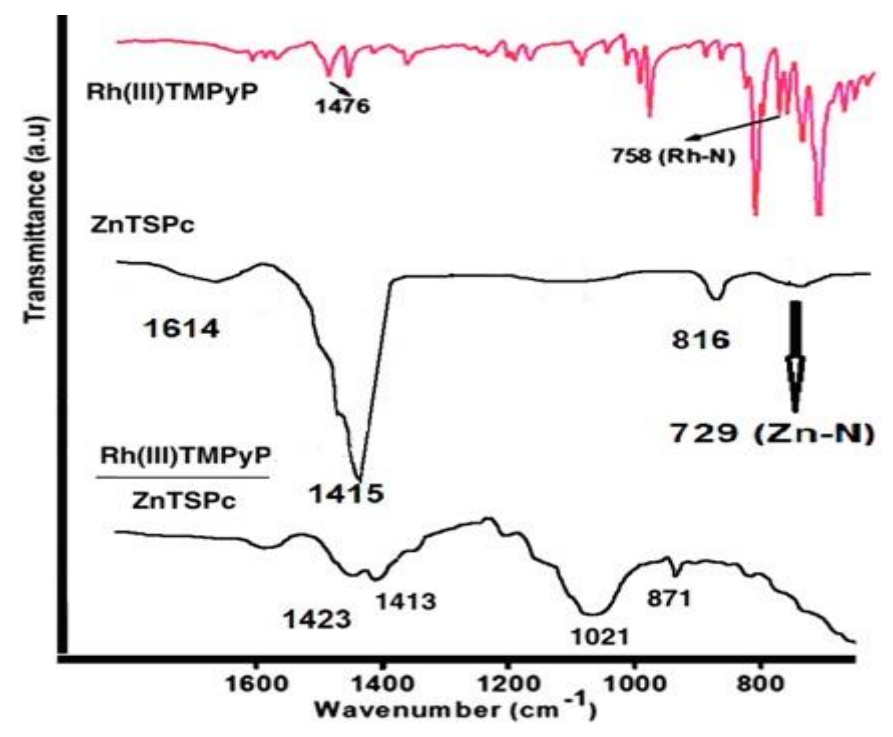

Fig. 5. IR spectra of RhTMPyP / ZnTSPc and individual components

The IR spectra of aromatic condensed benzene rings of metal phthalocyanines and porphyrins include the most extensive set of stretching and bending vibration bands: stretching vibrations of $\mathrm{C}-\mathrm{H}$ bonds (3046-3060 $\left.\mathrm{cm}^{-1}\right)$.

Relatively low intensity and skeletal C-C vibrations (1580-1610 $\left.\mathrm{cm}^{-1}\right)$ with intensities are varying from moderate to very low. The frequency of $1580 \mathrm{~cm}^{-1}$ is caused by stretching vibrations of the C$\mathrm{C}$ bond condensed with the pyrrole cycle.

Intense vibrations in the region of $947 \mathrm{~cm}^{-1}$ are totally symmetric stretching vibrations of $\mathrm{C}-\mathrm{C}$ bonds of the aromatic ring. Plane bending vibrations of $\mathrm{C}-\mathrm{H}$ are in the ranges of 1283-1289, 1158-1167, 10911096, and 1033-1060 $\mathrm{cm}^{-1}$. Out-of-plane bending vibrations of $\mathrm{C}-$ metal bonds of metal phthalocyanines and porphyrins appear in the range of $720-780 \mathrm{~cm}^{-1}$.

The complexity of the metallo-porphyrin molecule and the strong interaction of the ligand and metal ion could be responsible for the coupling of the ligand and metal-ligand vibrations [14, 15]. The high stability of the metallo-porphyrins suggests a somewhat higher frequency for the metal-nitrogen stretching mode. The far-infrared spectra of the metallo-porphyrins show a series of metal-dependent ligand absorption bands. The broad, medium intensity porphyrin deformation band at $500 \mathrm{~cm}^{-1}$ is shifted to a higher frequency in the metallo-porphyrins.

The most intense band in the far-infrared spectra of the metalloporphyrins appears at $350 \mathrm{~cm}^{-1}$, and the adjacent weaker band at $380 \mathrm{~cm}^{-1}$, can be attributed to the metal-free ligands and metal-nitrogen bond.

Vibrations of the $-\mathrm{C}=\mathrm{C}-\mathrm{N}=$ group (pyrrole fragment and nitrogen meso-atoms) appear as a low intensity band at $1396-1407 \mathrm{~cm}^{-1}$ and a very strong (characteristic of all phthalocyanines) band at 1320$1365 \mathrm{~cm}^{-1}$ [16].

At porphyrins, a high-intensity band at 1500 $1530 \mathrm{~cm}^{-1}$ corresponds to vibrations of nitrogen bridging atoms (meso-atoms -N=). Stretching vibrations including vibrations of the pyrrole and benzene residues are in the range of $1420-1480 \mathrm{~cm}^{-1}$; the band intensity varies from low to very high. The bands at $1594-1608 \mathrm{~cm}^{-1}$ and $1573-1582 \mathrm{~cm}^{-1}$ can be assign to C-N stretching vibrations, see Figure 5 .

Different from the IR spectroscopic method which only depends on the chemical composition and phase of the samples, the resonance Raman spectral features also depend on the excitation wavelengths. 


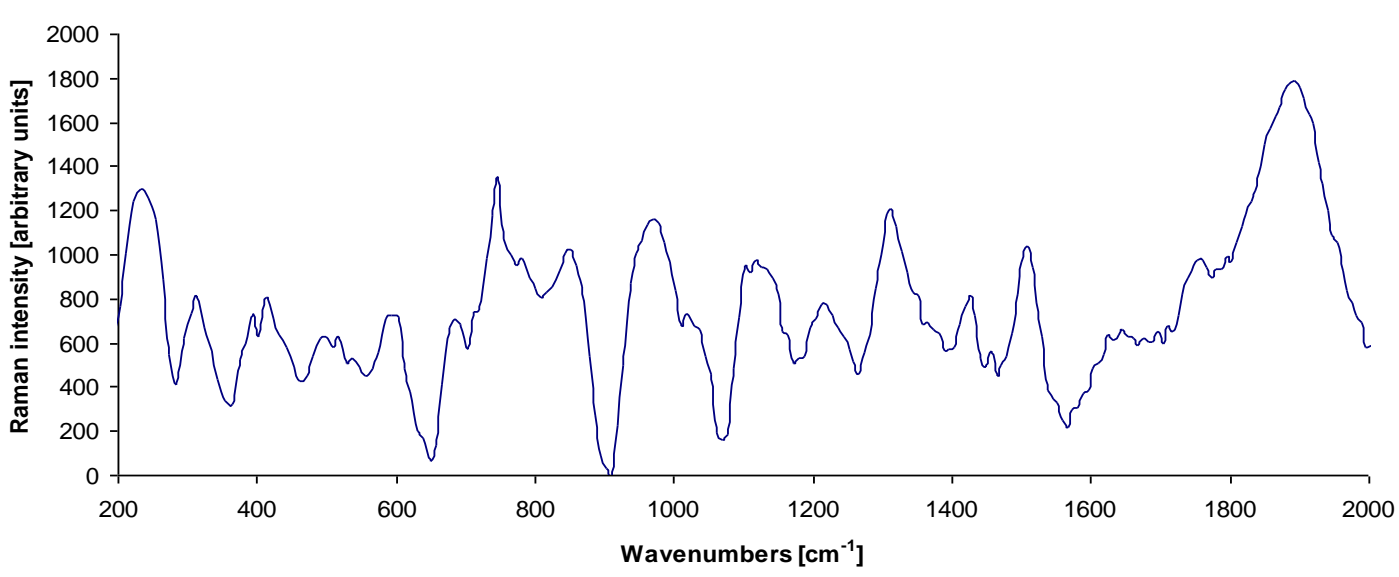

Fig. 6. Raman spectra of RhTMPyP / ZnTSPc

The obtained Raman spectrum shows prominent peaks whose Raman displacements are characteristic of the vibrational frequencies of the atoms in the molecules in which they are found, see Figure 6. The Raman spectra of analysed macro-heterocyclic compound shows some specific bands for these compounds at 1527 and $1538 \mathrm{~cm}^{-1}$. They have been collected using laser excitation sources emitting at 632.8 and $785 \mathrm{~nm}$. Under excitation at $632.8 \mathrm{~nm}$, the most intense Raman band appears at $1598 \mathrm{~cm}^{-1}$ which is assigned to the isoindole stretching.

Typical Raman marker bands of the monoanion radical Pc.- were observed at $1499-1512 \mathrm{~cm}^{-1}$ as a medium band and at $1521-1538 \mathrm{~cm}^{-1}$ as a band whose intensity increases with the aggregation, resulting from the coupling of $\mathrm{N}^{+}$with $\mathrm{SO}_{3}^{-2}$ respectively.

In the context of surface chemistry, porphyrins, phthalocyanines and their metal complexes stand out from other organic molecules because of their, in relation to size and complexity, remarkable stability, their specific ligand-functionality and reactivity towards metals, as well as the planar structures of their parent macrocyles [17].

\section{Conclusions}

To develop new sensitizers based on macroheterocyclic compounds, in this paper were studied the RhTMPyP/ ZnTSPc compound. Spectral properties of the studied materials indicated that these could be perspective for many applications, due to their versatility to change their central metal valence and to attach new substituents.

Under this context, RhTMPyP/ ZnTSPc is a special compound with proper properties. Their capacity of association has been identified by UVVis, FT-IR and Raman spectral techniques, which identified their internal bonds, their capacity to add new axial ligand and to create larger and versatile spatial structures.

\section{Acknowledgements}

The research leading to these results has received funding by Executive Agency for Higher Education, Research, Development and Innovation Funding (UEFISCDI) under the project PN-III-P11.2-PCCDI2017-0476 "New diagnosis and treatment technologies for the preservation and revitalization of archaeological components of the national cultural heritage", ctr. no 51PCCDI/2018.

\section{References}

[1]. Buchler J. W., Ng D. K. P., Metal Tetrapyrrole Double- and Triple-Deckers with Special Emphasis on Porphyrin Systems, New York, NY: Academic Press, 2000.

[2]. Bian Y., Zhang Y., Ou Z., Jiang J., Chemistry of sandwich tetrapyrrole rare earth complexes, in Handbook of Porphyrin Science, Singapore: World Scientific Publishing Co. Pte. Ltd., p. 249-460, 2011.

[3]. Ion R.-M., Yilmaz I., Bekaroglu O., Supramolecular assemblies of pyridyl porphyrin and diazadithia phthalocyanine, Journal of the Serbian Chemical Society, 64(7-8), p. 453-458, 1999.

[4]. Agirtas S., Ion R.-M., Bekaroglu O., Spectral study of the supramolecular assemblies porphyrins-phthalocyanines, Materials Science and Engineering: C, 7(2), p. 105-110, 1999.

[5]. Cook L. P., Brewer G., Wong-Ng W., Structural aspects of porphyrins for functional materials applications, Crystals, 7(7), p. 223-244, 2017.

[6]. Thompson S. J., Brennan M. R., Lee S. Y., Dong G., Synthesis and applications of rhodium porphyrin complexes, Chemical Society Reviews, 47(3), p. 929-981, 2018.

[7]. Suslick K. S., Rakow N. A., Kosal M. E., Chou J. H., The materials chemistry of porphyrins and metalloporphyrins, Journal of Porphyrins and Phthalocyanines, 4, p. 407-413, 2000.

[8]. de Toledo M., et al., Zinc phthalocyanine tetrasulfonateloaded polyelectrolytic PLGA nanoparticles for photodynamic therapy applications, Photodiagnosis and Photodynamic Therapy, $32,2020$.

[9]. Collman J. P., Garner J. M., Woo L. K., The chemistry of rhenium and tungsten porphyrin complexes in low oxidation states. Synthesis and characterization of rhenium and tungsten porphyrin 
dimers containing metal-metal multiple bonds, Journal of the American Chemical Society, 111 (21), p. 8141-8148, 1989.

[10]. Imahori H., Umeyama T., Kurotobi K., Takano Y., Selfassembling porphyrins and phthalocyanines for photoinduced charge separation and charge transport, Chemical Communications, 48(34), p. 4032-4045, 2012.

[11]. Pop S. F., Ion R.-M., Corobea M. C., Raditoiu V., Spectral and thermal investigations of porphyrin and phthalocyanine nanomaterials, Journal of Optoelectronics and Advanced Materials, 13(7), p. 906-911, 2011.

[12]. Logunov S. L., Rodgers M. A. J., Self-assembled ion-pair complexes between porphyrins and bipyridinium species: picosecond dynamics of charge recombination, The Journal of Physical Chemistry, 96(22), p. 8697-8700, 1992.

[13]. Giovannetti R., The use of spectrophotometry UV-Vis for the study of porphyrins, In: Jamal Uddin (Eds.), Macro to Nano Spectroscopy, p. 87-108, 2012.
[14]. Pratiwi R., Ibrahim S., Tjahjono D. H., Reactivity and Stability of Metalloporphyrin Complex Formation: DFT and Experimental Study, Molecules, 25, p. 4221-4228, 2020.

[15]. Olteanu L., Ion R. M., Teodorescu S., Stirbescu R. M., Dulamă I. D., Bucurică A. I., New metallo-porphyrins for solar energy conversion, Bulletin of the Transilvania University of Brasov, 10 (59), Series I, p. 47-54, 2017.

[16]. Aydin M., Akins D. L., Infrared and Raman Spectroscopic Characterization of Porphyrin and its Derivatives, In: Applications of Molecular Spectroscopy to Current Research in the Chemical and Biological Sciences, IntechOpen, 2016.

[17]. Georgescu R., Boscornea C., Calinescu I., State R., Raman IR and UV-Vis Spectroscopic Investigations of Some Substituted Phthalocyanines, Revista de Chimie (Journal of Chemistry), 66(10) p. $1554-1560,2015$ 